\title{
Investigating into the Culture Connotations of the Radical of Yu in the ShuoWenJieZi
}

\author{
Wang Xue \\ Department of Literature and Journalism, Inner Mongolia University
}

\begin{abstract}
As the first dictionary analyzing the structures of Chinese characters, explaining the meanings and identifying the pronunciations, the book ShuoWenJieZi (literally means discussing writing and explaining characters) compiled by Xu Shen in the Eastern Han Dynasty is of important significance in the Chinese language history as well as social language history. By means of researches on the section headers related to "fish" in the book, the development of both the fishery and fish food culture in the Qin and Han Dynasties can be discussed. Based on the section headers related to "fish" in the book ShuoWenJieZi, this paper analyzes the fishery development in the Qin and Han dynasties and examines the tradition of food culture reflected by the section headers related to "fish" as well as the prosperity and development of fish-eating culture.
\end{abstract}

Keywords: ShuoWenJieZi, the radical of Yu, cultural connotations

\section{Introduction}

As Edward Sapir once stated, "there is something behind the language and language cannot exist without culture. The socall culture is the summation of the habit and relief passed down, which can determine our living organization. " (Luo Changpei) The relationship between language and culture is permeating and blending with each other. On the one hand, language is an important part of human culture and a means to construct and inherit human culture; on the other hand, culture has a restrictive and decisive effect on language. It is an important content of cultural linguistics to explore culture through language.ShuoWenJieZi is the first book that systematically analyzes and studies the shape and the origin of Chinese characters.All together 103 characters were collected in the section headers related to "fish" which recorded a large number of fish names, body parts, tastes, features and fish foods. All these Chinese characters are not only the symbols to record language, at the same time, its meaning and contain rich cultural information, but also the important carriers of the fish culture in ancient China ${ }^{[1]}$.

In this paper, the radical of Yu in ShuoWenJieZi are taken as objects, and literature review methods, comparative mutual proof method, statistical induction method are used to conduct a comprehensive and in-depth study of the radical of Yu. By interpreting the section headers related to "fish" in ShuoWenJiezi, we can explore the fishery situation before the Qin and Han Dynasties as well as the development of fish food culture.

\section{Characters of Yu Radical and Fishery Development in Qin and Han Dynasties}

The numerous rivers and lakes, as well as the abundant marine resources in our country have provided superior natural conditions for fishery development. ShuoWenJieZi has recorded "Yu (澳)" that refers to the action of fishing. The character refers to the aquatic animal was originally the same as the activity of fishing, with a homologous relationship. By analyzing characters of $\mathrm{Yu}$ radical in ShuoWenJieZi, it can be seen that China's fishery industry during the Qin and Han Dynasties has achieved significant development, which is mainly manifested in two aspects: the deepening of people's understanding of fish and the improvement of fishing and utilization.

\subsection{A Deepened Understanding of Fish}

Human's understanding of fish has gone through a process from vague to clear, from disordered to systematic throughout the Pre-Qin and Han Dynasties, and got deepened due to the ancient fisheries development. This is firstly reflected in their deepening acquisition of fish diversity. This paper compared the number of the fish names collected in "The Book of Songs" and "Er Ya" with the characters with $\mathrm{Yu}$ in ShuoWenJieZi, so as to investigate the development of human's cognition of fish species in the Qin and Han Dynasties ${ }^{[2]}$.

"The Book of Songs", the first collection of poems in China, was written in the early Western Zhou Dynasty and the middle Spring and Autumn Period. According to the statistics by Cheng Qingtai, a fish expert, there are 13 fish names recorded in this Book. "Er Ya" was roughly written in the end of the

[Received 01 June 2020; Accepted 21 Dec 2020; Published (online) 31 Dec 2020] Publisher's Note: RCLSS stays neutral regard to jurisdictional claims published maps (c) (1) 
Warring States Period and the early Western Han Dynasty, slightly later than the "Book of Songs". One of its chapter, "Shi Yu", has recorded the names of some fish, amphibians and other aquatic animals, including 39 fish names based on the author's statistics. While, the number of fish names increased swiftly to 76 according to the author's statistics in the Eastern Han Dynasty when ShuoWenJieZi was written ${ }^{[3]}$. It can be seen from the above records that the number of fish names was in an uptrend. This not only reflected the increase in fishing output during the Qin and Han Dynasties, but also indicated that people had a more in-depth and detailed understanding of fish species than the past. For instance, crucian and carp are Cyprinidae fish with very similar body appearance of being flat and abdomen color, but were named separately in ShuoWenJieZi, indicating that people has recognized their characteristics and differences of these fish. In the explanation of fish names in ShuoWenJieZi, people's recognition of physical characteristics or life habits for specified fishes in Qin and Han Dynasties is highlighted through the detailed interpretations of some fish in addition to the explanation of "fish names" by categories. In comparison with "The Book of Songs" and "Er Ya", ShuoWenJieZi strives to give a comprehensive introduction to fish from the aspects of color, posture, habits, and place of origin. From this point of view, ShuoWenJieZi has enriched the description of fish in previous works ${ }^{[4]}$.

\subsection{Fishing and Utilization of Fish}

The ancients continuously sum up experience from long-term fishing practices to further improve the shipping techniques with more abundant methods.

In the Qin and Han dynasties, bamboo woven cages have been used to catch swimming fish. This kind of fishing gear is called "罩"(zhao) and "笴"(gou). ShuoWenJieZi explained that: "罩(zhao) is a kind of fishing gear. 罩(zhao) is a fishing bamboo cage in cylindrical shape with smaller size at the top and bigger size at the bottom. Fish cannot swim out after entering into the cage. It looks like a chicken cage. The fisherman fetches the fish by pressing the water with hands." The fish caught are not only for food, but also used as decoration. According to the ShuoWenJieZi record, the 鰅 (yu) fish was caught and handed over to the 考工令 (kaogongling) for weapon decoration with the skin of the fish. ShuoWenJieZi: 鰅(yu), is a kind of fish name with colorful skins from 乐浪(lelenag) and 东暆(dongyi). It is firstly caught by 输考工(shukaogong) in the fourth year of Emperor Xuan of Han. Yangzhou offered the 鰅(yu) during the reign of King Cheng of Zhou Dynasty." 输考工(shukaogong), also named 考工令 (kaogongling), is the officer in charge of weapon manufacturing. Not only the the skin of 鰅(yu) fish but also the 鲛(jiao) fish can be used for decorating the swords and other weapons. ShuoWenJieZi: "鲛(jiao), is a kind of sea fish whose skin can be used to decorate sword." "Huainanzi . interpretation on mountain": "There could be two 鲛(jiao) in the same deep pool". Gaoxiu explained: "鲛(jiao), is a long fish with shining skin. It is considered to decorate the mouth of sword.". These are the records that the skin of 鲛(jiao) is used to decorate weapons.

3.Character of Yu Radical and Fish-eating Culture Evolution 3.1 Legacy of Fish-eating Habitude

ShuoWenJieZi: "鱻(xian), is the meaning of fresh fish. This character stands for three fresh fishes rather than spoiled fished." 鱻(xian) is the original character to express the meaning of " fresh" and later used in the phonetic loan character 鲜(xian). During the ancient times when floods happened frequently, fish was of great significance to people's production and life as the main food of the ancestors. The character "新鲜"(xin xian) is composed of three 鱼(yu), and therefore means fresh and delicious because of many fishes, which reflects the relics of life where fish is the main food. It is a sentence from "Mencius" that "Fish is my favorite; Bear's paw is also my favorite". The significant value of fish is embodied apparently since it is put in the equal position as bear's paw.

\subsection{Prosperity of Fish Food Culture}

The Chinese nation has a long history of food culture. People not only use fish to meet basic survival needs, but also create a great diversity of fish-eating manners to promote the prosperity and development of fish-eating culture. The words such as "鮨"(pronounced as Yi) and "善"(pronounced as Zha) are correspondently created.

Since fish is easy to get putrid and deteriorate, people process and store them to save for future consumption. It is an effective approach to extend the edible period of validity by making fish into sauces. ShuoWenJieZi: "鮨(Yi) is a kind of fish sauce from central Sichuan. The character 鮨(Yi) is composed of the radicals of 鱼(yu) and 旨(zhi). It is also called 鲅(wei) in another saying". The fish sauce, 鮨(Yi), is made from fish meat and marinated in salt and vinegar ${ }^{[5]}$.

Select fish or meat as raw materials, first process and cut them into small pieces, then marinate them with salt, liquor, and spices and put them in a container layer by layer. The special processed rice shall be scattered between each layer of fish and meat. The 鱽(zha) is therefore prepared after sealing the container and placing it aside for some time. It is cited from ShuoWenJieZi that "善(zha) is a type of fish from QinghaiTibet Plateau. It is called 䰼(qin) in the south and 善(zha) in the north. ${ }^{[6] "}$

\section{Conclusion}

To summarize what has been mentioned above, ShuoWenJieZi has not only recorded the interpretation of the structures, pronunciations and meanings of Chinese characters in traditional literature, but also saved a large amount of data about the social lives, science and technology, ideology and culture in ancient times which fully reflect the 
cultural information in material production, social systems and spiritual culture of our ancestors. characters of Yu radical in ShuoWenJieZi eflected the further understanding on fish by our ancestors, the progress of the fishery activities and the development of fishery production during the Qin and Han Dynasties. The resulting prosperity and development of fish food culture is also fully demonstrated by the section headers related to "fish" in ShuoWen Jiezi. Our ancestors used to live on eating raw fish, then developed a variety of fish food cooking methods, such as "mashed fish", "pickled fish" and "salted fish", which not only enriched people's daily diet but also greatly expanded and promoted the profound Chinese food culture. With the increasing popularity of ShuoWen Jiezi, we can make further cultural researches on the section headers related to "fish" in the book, so that the relics of ancient fish culture will be explored by the more scholars.

\section{References}

[1] Luo, C. (2004). Language and Culture. Beijing: Beijing Publishing House. pp. 3.

[2] Xu, S., (2013). ShuoWenJieZi. Beijing: Zhonghua Book Company, pp.242-245.

[3] Guo, P. (2006). Er ya. Beijing: Beijing Book Publishing House.

[4] Dai, Z. (1996). Introduction to Cultural Linguistics. Beijing: Chinese Press pp.14.

[5] Lou, L., (2012). A look at the ancient Chinese fish cunture in the Radical of Yu in the ShuoWenJieZi. Journal of Zhejiang Ocean University, Phase V.

[6] Cheng, Q., (1984). Interpretation of fish recorded in ShuoWenJieZi. Nature science magazine, Phase IV. 